\title{
sciendo
}

\section{Effect of letrozole on spermogram parameters and hormonal profile in infertile men: A clinical trial study}

\author{
Sepideh Peivandi ${ }^{1}$, Hamed Jafarpour ${ }^{2}$, Mina Abbaspour ${ }^{1}$, Aghdas Ebadi ${ }^{1}$ \\ ${ }^{1}$ Department of Obstetrics and Gynecology, Faculty of Medicine, Mazandaran University of Medical Sciences, Sari, Iran; \\ ${ }^{2}$ Student Research Committee, Faculty of Medicine, Mazandaran University of Medical Sciences, Sari, Iran \\ E-mail:dr.jamkhane@gmail.com
}

Objective. There is no reliable treatment for men with idiopathic infertility, but the relationship between severe sperm production and the ratio of estrogen to testosterone levels has been shown. Aromatase is an enzyme that plays an important role in converting testosterone to estradiol and androstenedione to estrogen. Aromatase inhibitors can increase testosterone and androgen production without increasing the amount of estrogen in circulation. The aim of this study was to evaluate the effect of aromatase inhibitor letrozole on the male infertility.

Method. This pre- and post-quasi-experimental clinical trial was carried out on 41 men with an infertility diagnosis. The basic hormonal profile included FSH, LH, testosterone, and estradiol. The ratio of testosterone to serum estradiol was also calculated and recorded. The sperm analysis was performed before the treatment and the seminal parameters were evaluated and recorded. Patients were then treated with letrozole $2.5 \mathrm{mg}$ daily for 4 months. At the end of 4 th months, the hormonal profile was studied and seminal analysis performed and recorded.

Results. The levels of FSH, LH, testosterone, and estradiol, and the ratio of testosterone to estradiol increased significantly after letrozole treatment. The sperm concentration, sperm motility, and sperm forward motion significantly increased after letrozole treatment. Sperm morphology only lightly altered.

Conclusion. The ratio of testosterone to estradiol levels in infertile men treated with aromatase inhibitor improved and caused changes in sperm parameters. Letrozole may be used to improve sperm parameters in infertile men with low serum testosterone to estradiol ratio.

Key words: male infertility, aromatase inhibitor, testosterone, estradiol, letrozole

Aromatase is a cytochrome P450 enzyme that plays a role in converting testosterone to estradiol and androstenedione to estrogen. This enzyme is also found in the reproductive organs of women, adipose tissue, in the testis, liver, brain, and other tissues (Inkster et al. 1995). In the testis, aromatase is found in Leydig and Sertoli cells also found in germ cell tumors. Aromatase inhibitors are able to increase production of testosterone and estrogen without increasing the circulating estrogens levels, unlike estrogen receptor modulators (Pavlovich et al. 2001).
Aromatase inhibitors interact with the aromatase in estrogen secreting tissues, thus limiting the estrogen production by maintaining the level of testosterone. Finally, aromatase inhibitors can be effective in a variety of clinical conditions, including breast cancer treatment, endometriosis, endometrial, breast, and disorder in spermatogenesis (Casper and Mitwally 2006). About 50 years ago, the first generation of aromatase inhibitors was known, but since a decade, the therapeutic use has been practiced. The first generation of these drugs had many complica-

Corresponding author: Aghdas Ebadi, M.D., Department of Obstetrics and Gynecology, Faculty of Medicine, Mazandaran University of Medical Sciences, Sari, Iran; phone: +989111532890; e-mail: dr.jamkhane@gmail.com. 
tions, but by the production of the third generation, these complications were controlled that led to the successful treatment of estrogen-dependent diseases (Buzdar and Howell 2001). Various aromatase inhibitors have been used in the treatment of male infertility and studies have been reported with successful results in limited number patient with idiopathic oligozoospermia and non-obstructive azoospermia related to the T/E2 ratio (Clark and Sherins 1989). The $\mathrm{T} / \mathrm{E} 2$ ratio levels in infertile men treated with aromatase inhibitors have been improved and changed sperm parameters. Research has shown that infertile men with low serum estradiol levels can be treated with aromatase inhibitors. Treatment increases the T/E2 ratio along with the increase of sperm parameters (Haider et al. 2007). Letrozole is an aromatase inhibitor attached to cytochrome P-450, type 2 and the third generation that inhibits estrogen biosynthesis reversibly (Haider et al. 2007). Femara is the trade name of aromatase inhibitor act as anti-cancer, which reduces estrogen production. The drug is available in $2.5 \mathrm{mg}$ tablets and well absorbed after oral administration (with a high bioavailability of $99.9 \%$ ) and the impact of food on its absorption is poor. It has wide and fast distribution and about $60 \%$ of it binds to plasma proteins, excreted mainly urinary $(90 \%)$ and with a half-life of about 2 days (Smith and Norton 1998). Letrozole is a non-steroidal aromatase inhibitor that has been introduced as the hormone responsible for the treatment of breast cancer. Estrogen is converting to androgen by aromatase. Letrozole prevents the formation of estrogen with a reversible competitive mechanism that binds to the cytochrome P450. The function of the drug is specific and does not reduce the production of mineralocorticoids or corticosteroids. The anti-estrogenic action of tamoxifen, the main drug before the introduction of aromatase inhibitors, due to its interaction with the estrogen receptor, mainly inhibits estrogen production. Letrozole has been shown to reduce estrogen levels by up to $98 \%$ by elevating testosterone level (Clark and Sherins 1989).

In human studies, objectives have been focused on the use of aromatase inhibitors in infertile men with low levels of testosterone and an abnormal T/E ratio. Given that in some studies, aromatase inhibitors have been reported to be ineffective in the treatment of infertility (Patry et al. 2009), and still they are used in infertility in the IVF centers in Iran. Therefore, in this study, the effect of aromatase inhibitors on male infertility in the infertile referring to the infertility clinic of Imam Khomeini Hospital in Sari was studied.

\section{Materials and methods}

Subjects. This quasi-experimental before and after clinical trial was conducted on the men referring to the infertility center of Imam Khomeini Hospital in Sari in 2013-2014 with a diagnosis of male infertility. The study was approved by the ethics committee of Mazandaran University of Medical Sciences (ethics committee registration code: 13/12/93). Inclusion criteria for infertile men with abnormal parameters of semen fluid were based on WHO criteria, which were serum T/E2 ratio $<10$ (testosterone $\mathrm{mg} / \mathrm{dl}$ and estradiol $\mathrm{pg} / \mathrm{ml}$ ). The patients were explained about the method of the study and written consent was obtained from them.

According to the previous studies (Saylan et al. 2011; Gregoriou et al. 2012) on the efficacy of letrozole in the treatment of infertile men and considering the differences in hormonal (estradiol) and seminal (number, motility, and concentration) findings and considering the probability of type 1 error $=0.05$ and as a single domain (improvement after treatment), the highest sample size was 45 patients. With a $20 \%$ drop, the final sample size was considered to be 55 .

Parameters. The height, weight, and BMI of patients were measured by the standard method and recorded in the information form. Basic hormonal profiles, such as FSH, LH, testosterone, and estradiol were measured and recorded in the information form.

Hormonal profile was assayed using $\mathrm{ROCH}$ kit with ELECSYS instrument (made in Iran, 2010) by Electrochemomagnetic method. The ratio of testosterone to serum estradiol was also calculated and recorded. The baseline sperm analysis was performed before the treatment and the seminal parameters were recorded based on the World Health Organization (WHO) criteria. Semen sample was obtained through the masturbation; the semen was poured into a clean container and studied within one hour. The patients were instructed not to have sex 2-3 days prior to the sampling, but there was no restriction of having sex during the time of letrozole administration. After obtaining consent, the subjects were treated with an aromatase inhibitor, letrozole, and $2.5 \mathrm{mg}$ daily for 4 months. At the end of 4 months, the hormonal profile and seminal analyses were re-evaluated and recorded in the information form. Infertile men with obstructive azoospermia, acute and chronic inflammatory diseases, varicocele, taking fertility medications, such as metformin were excluded from the study. The patients under oral or intravenous fertility medication in the recent 2 months were excluded. 
Statistical analysis. Paired t-test was used in comparing the quantitative outcome variables before and after treatment with letrozole. All analyzes were performed on a single domain (improvement after the treatment) and considering the probability of type 1 error $=0.05$. Statistical analysis was performed using the Stata version 10 software.

\section{Results}

In this study, 41 men with different spermogram and hormonal parameters were examined before and after treatment with letrozole. The age of the subjects was 33 to 51 years (mean and standard deviation of 41.22 \pm 4.78 ) and their BMI varied from 20.06 to 34.94 (mean and standard deviation of $27 \pm 3.97$ ) (Table 1).

The FSH and LH levels in patients before intervention were $5.74 \pm 1.71 \mathrm{mIU} / \mathrm{ml}$ and $5.07 \pm 1.89 \mathrm{mIU} / \mathrm{ml}$, respectively; and after intervention, increased to $7.42 \pm 1.6 \mathrm{mIU} / \mathrm{ml}$ and $6.62 \pm 1.8 \mathrm{mIU} / \mathrm{ml}$, respectively $(\mathrm{p}<0.001)$ (Table 2).

Testosterone (T) level before intervention was $25.15 \pm 11.1 \mathrm{ng} / \mathrm{ml}$ and significantly increased to $40.73 \pm 12.6 \mathrm{ng} / \mathrm{ml}$ after intervention $(\mathrm{p}<0.001)$. Blood estradiol level (E2) before and after intervention was $4.8 \pm 1.66 \mathrm{pg} / \mathrm{ml}$ and $5.99 \pm 1.51 \mathrm{pg} / \mathrm{ml}$, respectively, $(\mathrm{p}=0.002)$. The T/E2 ratio after $(7.09 \pm 2.35)$ and before $(5.89 \pm 2.84)$ intervention was statistically significant $(\mathrm{p}=0.014)$ (Table 2).

The spermogram parameters and their comparison before and after treatment are given in Table 3 and show that the semen volume, sperm concentration, sperm motility, and forward motion increased. However, this increase was statistically significant for sperm concentration, sperm motility and forward motion $(p<0.001)$. We observed only light sperm morphology alterations (Table 3 ).

\section{Discussion}

The aim of this study was to evaluate the effect of aromatase inhibitors on male infertility. The levels of FSH, LH, testosterone, and estradiol, and the ratio of testosterone to estradiol, increased significantly after treatment. The semen volume, sperm concentration, sperm motility, and sperm forward motion increased, statistically significant in sperm concentration, sperm motility, and sperm forward motion. Morphology decreased, but statistically insignificantly.

Recent studies have identified a potential endocrine disorder in male infertility. In some men, the relation of severe sperm production disorder to the
Table 1

Demographic information of patients

\begin{tabular}{lcc}
\hline Variable & Variation range & Mean \pm S.D. \\
\hline Age of patients & $33-51$ & $4.78 \pm 41.22$ \\
BMI & $20.06-34.94$ & $3.95 \pm 27.00$ \\
\hline
\end{tabular}

Table 2

Hormonal parameters of patients before and after treatment

\begin{tabular}{lccc}
\hline Hormonal parameters & $\begin{array}{c}\text { Before } \\
\text { intervention }\end{array}$ & $\begin{array}{c}\text { After } \\
\text { intervention }\end{array}$ & p-value \\
\hline FSH $(\mathrm{mIU} / \mathrm{ml})$ & $1.71 \pm 5.74$ & $1.6 \pm 7.42$ & $<0.001$ \\
$\mathrm{LH}(\mathrm{mIU} / \mathrm{ml})$ & $1.89 \pm 5.07$ & $1.8 \pm 6.62$ & $<0.001$ \\
Testosterone $(\mathrm{T})(\mathrm{ng} / \mathrm{ml})$ & $11.1 \pm 25.85$ & $12.6 \pm 40.73$ & $<0.001$ \\
Estradiol $(\mathrm{E} 2)(\mathrm{pg} / \mathrm{ml})$ & $1.66 \pm 4.8$ & $1.51 \pm 5.99$ & 0.002 \\
T/E2 & $2.84 \pm 5.89$ & $2.35 \pm 7.09$ & 0.014 \\
\hline
\end{tabular}

Table 3

Parameters of spermograms of patients before and after treatment

\begin{tabular}{lccc}
\hline $\begin{array}{l}\text { Spermogram } \\
\text { parameters }\end{array}$ & $\begin{array}{c}\text { Before } \\
\text { intervention }\end{array}$ & $\begin{array}{c}\text { After } \\
\text { intervention }\end{array}$ & p-value \\
\hline Semen volume $(\mathrm{ml})$ & $0.57 \pm 2.07$ & $0.57 \pm 2.15$ & n.s. \\
$\begin{array}{l}\text { Sperm concentration } \\
\text { (number/ml) }\end{array}$ & $6.99 \pm 21.46$ & $9.29 \pm 28.44$ & $<0.001$ \\
\hline Sperm motility (\%) & $5.04 \pm 22.59$ & $7.93 \pm 31.76$ & $<0.001$ \\
\hline $\begin{array}{l}\text { Forward motion }(\%) \\
9.18 \pm 20.95\end{array}$ & $9.63 \pm 29.76$ & $<0.001$ \\
\hline Morphology $(\%)$ & $2.89 \pm 7.83$ & $2.26 \pm 7.32$ & n.s. \\
\hline
\end{tabular}

ratio of estrogen to testosterone level has been shown (Carreau et al. 2012; Pavlovich et al. 2001). Estrogen is produced from androgen by the activity of aromatase (Clark and Sherins 1989). On the other hand, animal studies have shown that the local aromatase is essential for spermatogenesis and pointing the role of estrogen in the development of male germ cell (Carreau et al. 2012).

It has been shown that estrogen is essentially important factor in fluid reabsorption in the rats testicle, and lack of sufficient fluid absorption increases the intratesticular pressure, resulting in a testicular atrophy in mice (Wang et al. 1985). Therefore, in the animal models, the effect of estrogen inhibitors is not well known. While the probable potential devastating the role of estrogen on the germinal epithelium has been shown. Also, high levels of estrogen accompanied by low-level androgen may lead to spermatogenesis disorder (Bharti et al. 2013).

In human studies, the objectives have been focused on the use of aromatase inhibitors in infertile men 
with low levels of testosterone and an abnormal T/E ratio. In some studies, aromatase inhibitors have been reported to be ineffective in the treatment of infertility (Patry et al. 2009) and still, this drug is used in the IVF Centers in Iran, as a selective drug in the treatment of infertile men. Therefore, the effect of aromatase inhibitors on the male infertile was studied. Their mean age was at the onset of the middle age. Data on the effect of age on fertility is unclear, but in men with an increase of age, the level of testosterone declines, while the serum estradiol and estrogen levels increase. Studies have shown that aging in men reduces sperm density (Ng et al. 2004).

BMI had an extensive range, with a mean of about 27. It was observed that the ratio of T/E2 was significantly lower among overweight men (BMI $>25$ ) compared to men with lower BMI. It has also been found that men with higher BMI had changed their sperm quality (Fejes et al. 2006). The levels of FSH, $\mathrm{LH}$, testosterone, and estradiol, as well as the ratio of T: E2 increased significantly after treatment $(\mathrm{p}<0.05)$. It corresponds with the data given by Raman and Schlegel (2002) in a case-control study, giving daily 100 to $200 \mathrm{mg}$ test lactone and /or daily $1 \mathrm{mg}$ anastrozole to infertile men in the United States (Raman and Schlegel 2002).

Gregoriou et al. (2012) on their study about the hormonal changes and seminal parameters following treatment with aromatase inhibitors in the infertile men with low T/E ratios, have found similar results and have shown that use of aromatase inhibitors in infertile men with low T/E ratios improves hormonal and seminal parameters (Gregoriou et al. 2012). Saylam et al. (2011) in a clinical trial, have examined the effect of letrozole aromatase inhibitor on BMI, hormonal parameters and sperm of infertile men, the findings corresponded to our data. The use of letrozole in infertile men with a low T/E2 ratio could be effective in improving hormonal and sperm parameters (Saylam et al. 2011).

In our study, the parameters of the spermogram and their comparison before and after treatment were also carried out, in that the increase in the semen volume, sperm concentration, sperm motility and sperm forward motion was noticed that was statistically significant $(\mathrm{p}<0.001)$ in sperm concentration, sperm movement and sperm forward motion, but with the insignificant difference in case of semen volume. We noticed a decrease in morphology, which was statistically insignificant. In the case of control study, Raman and Schlegel (2002) have used a test lactone and/ or anastrozole, to increase of sperm concentration in semen (from 5.5 million to 11.2 million per $\mathrm{ml})$, increase of motility (14.7\% to $21 \%)$ and normal morphology (6.5\% to $12.8 \%$ ), which agreed with our findings in sperm concentration and motility, but disagreed with the morphology data (Raman and Schlegel 2002).

In a clinical trial conducted by Clark and Sherins (1989) in Georgia, the effect of treatment with aromatase inhibitors on idiopathic oligospermia has been studied. In this study, 25 patients with idiopathic oligospermia were treated with $2 \mathrm{~g} /$ day test lactone and/or placebo for 8 months. Total estradiol and testosterone levels during the treatment with testolactone did not change at a baseline level and in the placebo group. Although the free testosterone levels increased by $36 \%(p<0.01)$, the free estradiol level increased insignificantly. Also, LH and FSH levels increased by $15 \%$ and $20 \%$, respectively $(\mathrm{p}<0.05)$, and the 17-alpha-hydroxyprogesterone level increased by $90 \%(\mathrm{p}<0.05)$.

Sperm parameters remained unchanged during treatment with testolactone and placebo and no pregnancy occurred during the 16 months follow up. These data have shown that long-term administration of testolactone was not effective in the treatment of infertility due to idiopathic oligospermia (Clark and Sherins 1989). The findings of the above study agree with our data on the hormone parameters, but differed from seminal parameters that could be related to the spermogram laboratory techniques and the use of testolactone.

Letrozole is 20 times more potent in inhibiting the aromatase enzyme than other drugs. The third generation has a greater estrogen inhibition than the first and second generations, which is a thousand times more potent. The third generation of aromatase inhibitors, in contrast to the previous two generations, acts selectively and does not interfere with the secretion of glucocorticoids, mineralocorticoids, and/or thyroxine secretion (Smith and Norton 1998). These drugs are orally aborted properly with quick distribution in the body a half-life of about two days and reach a constant serum level after one week (Haynes et al. 1991).

In the case of male infertility, various aromatase inhibitors have been used, and studies have reported successful results in limited numbers of patients with idiopathic oligozoospermia and nonobstructive azoospermia in relation with the T/E2 ratio (Clark and Sherins 1989). However, few studies have been conducted on the effect of letrozole on the treatment of male infertility in relation to the reduction of the T/E2 ratio. According to the findings of this study and review of the other studies, the mechanism of 
action of letrozole in the treatment of male infertility is preventing the formation of estrogen with a reversible competitive mechanism that binds the cytochrome P450 (Haider et al. 2007).

Though the increase of sperm production and sperm motility stimulation by medication, such as the use of estrogen receptor modulators, that is clomiphene and tamoxifen citrate have been shown. These drugs increase the level of testosterone in the testicle and stimulate the production of FSH that led to the increase of estrogen level (Inkster et al. 1995). In contrast, the estrogen receptor modulators that were the main drugs prior to the introduction of aromatase inhibitors, more inhibit the estrogen production due to their interaction with the estrogen receptor. It has been shown that Letrozole reduces estrogen levels by up to $98 \%$ by increasing testosterone levels (Smith and Norton 1998). Our study showed that the ratio of testosterone to estradiol was improved in infertile men under treatment with aromatase inhibitors that changes the sperm parameters. Data show that infertile men with a low serum T/E2 ratio can be treated with aromatase inhibitors. With treatment, an increase in T/E2 ratio occurs with the increase of sperm parameters (Smith and Norton 1998). Limitation of this study is the lack of control group. Since this is not a comparative investigation, we only studied the patients seeking treatment. For more effective, relevant studies, further studies with the control group recommended.

\section{Conclusion}

In summary it can be said that letrozole, as an aromatase inhibitor, may be used effectively to improve sperm parameters in infertile men with low serum T/E2. Fertility potential may be achieved in oligospermia men after treatment. In addition, letrozole with a relative effect on infertile men with non-obstructive azoospermia and by improving the sperm-related factors facilitates the fertility in these men.

\section{Acknowledgments}

The authors would like to acknowledge the Deputy of Research and Technology at Mazandaran University of Medical Sciences who provided the funding for this study. The study has been approved by the ethics committee of Mazandaran University of Medical Sciences (ethics committee registration code: 13/12/93).

\section{References}

Bharti S, Misro M, Rai UJ. Clomiphene citrate potentiates the adverse effects of estrogen on rat testis and downregulates the expression of steroidogenic enzyme genes. Fertil Steril 99, 140-148, 2013.

Buzdar A, Howell A. Advances in aromatase inhibition: clinical efficacy and tolerability in the treatment of breast cancer. Clin Cancer Res 7, 2620-2635, 2001.

Carreau S, Bouraima-Lelong H, Delalande C. Estrogen, a female hormone involved in spermatogenesis. Adv Med Sci 57, 31-36, 2012.

Casper RF, Mitwally MF. Review: aromatase inhibitors for ovulation induction. J Clin Endocrinol Metab 91, 760-771, 2006.

Clark RV, Sherins RJ. Treatment of men with idiopathic oligozoospermic infertility using the aromatase inhibitor, testolactone. Results of a double-blinded, randomized, placebo-controlled trial with crossover. J Androl 10, 240-247, 1989.

Fejes I, Koloszar S, Zavaczki Z, Daru J, Szollosi J, Pal A. Effect of body weight on testosterone/estradiol ratio in oligozoospermic patients. Arch Androl 52, 97-102, 2006.

Gregoriou O, Bakas P, Grigoriadis C, Creatsa M, Hassiakos D, Creatsas G. Changes in hormonal profile and seminal parameters with use of aromatase inhibitors in management of infertile men with low testosterone to estradiol ratios. Fertil Steril 98, 48-51, 2012.

Haider H, Solanki KS, Pal G, Singh MK, Kothari JS, Agarwal VK. Process for the preparation of letrozole. WO/2007/054964, PCT/IN2006/000337, 2007. https://patentscope.wipo.int > search > detail.

Haynes BP, Jarman M, Dowsett M, Mehta A, Lonning PE, Griggs LJ, Jones A, Powles T, Stein R, Coombes RC. Pharmacokinetics and pharmacodynamics of the aromatase inhibitor 3-ethyl-3-(4-pyridyl)piperidine-2,6-dione in patients with postmenopausal breast cancer. Cancer Chemother Pharmacol 27, 367-372, 1991.

Inkster S, Yue W, Brodie A. Human testicular aromatase: immunocytochemical and biochemical studies. J Clin Endocrinol Metab 80, 1941-1947, 1995.

Ng KK, Donat R, Chan L, Lalak A, Di Pierro I, Handelsman DJ. Sperm output of older men. Hum Reprod 19, 1811$1815,2004$. 
Patry G, Jarvi K, Grober ED, Lo KC. Use of the aromatase inhibitor letrozole to treat male infertility. Fertil Steril 92, 829.e1-829.e2, 2009.

Pavlovich CP, King P, Goldstein M, Schlegel PN. Evidence of a treatable endocrinopathy in infertile men. J Urol 165, 837-841, 2001.

Raman JD, Schlegel PN. Aromatase inhibitors for male infertility. J Urol 167, 624-649, 2002.

Saylam B, Efesoy O, Cayan S. The effect of aromatase inhibitor letrozole on body mass index, serum hormones, and sperm parameters in infertile men. Fertil Steril 95, 809-811, 2011.

Smith IE, Norton A. Fadrozole and letrozole in advanced breast cancer: clinical and biochemical effects. Breast Cancer Res Treat 49, S67-S77, 1998.

Wang JM, Gu CH, Tao L, Wu XL. Effect of surgery and efferent duct ligation on testicular blood flow and testicular steroidogenesis in the rat. J Reprod Fertil 73, 191-196, 1985. 